\title{
Smart System for Efficient Monitoring of Food Grains
}

\author{
Kavitha D, Anusha R, Deepa Lakshmi V, Mythili N, Shali A
}

\begin{abstract}
Agriculture is the main source of Indian economy. $16 \%$ of total GDP is depends on agriculture. So, maintaining a proper environment for storing the food grains is important. The food grains are stored in the silos. It is required a automated system which is used for monitoring the quality attributes of agricultural and food products. For increasing the productivity the food industry uses pests which affects the health of the consumers. Here a sensor based monitoring system which maintains the temperature, humidity and a co2 sensor is used to detect the presence of the insects and ultrasonic sensor keeps away the insects from the food grains and also avoids the usage of pests. The information is stored in the cloud and is passed to the mobile device of the silos incharge.
\end{abstract}

IndexTerms-Agriculture,FoodGrain Storage, Wireless network,Sensors,IOT,Cloud.

\section{INTRODUCTION}

One-third of the food produced annually for human consumption is wasted is in itself unconscionable in a world where population of 870 million, or one in eight people, go hungry every day. A United Nations Food organization and Agriculture Organization report now displays that this high volume of wastage that occurs right through the chain of food supply exert an adverse impact on nature that is land, water, biodiversity and climate change.

To increase the productivity of food grains, a automated system is designed for monitoring and controlling of the grain condition. The Arduino system with SCM is a lower machine control unit which monitor the humidity, temperature, ultrasonic, $\mathrm{Co} 2$, moisture data. Then the data is processed and the details of the grain condition are predicted based on the neural network.
A computer system is said to be an embedded system when a dedicated function within an electrical system or a larger mechanical system, with a real-time computing constraints. Almost $98 \%$ of all microprocessors which are manufactured are used in embedded systems In this method the condition of the grain storage details such as temperature, humidity and the data is collected and scanned by multi-sensor. The data is then processed via multi-regional information fusion and the data is stored on cloud via Ethernet. This method aims to reduce the wastage of food grains during storage.

\section{PROPOSED SYSTEM AND RELATED ARCHITECTURE}

The food grains which are stored in the silos needs to be monitored continuously for keeping it fresh and protect from insects without using any harsh chemicals or pests. This monitoring of stored grains in the silos can be made automated with the help of sensors which are connected to Arduino and are transferred to the monitoring unit with the help of cloud.

The data collected from grain environment such as humidity and temperature are stored by DHT11-sensor. If the Humidity level is high automatically fan will rotate. At the same time corresponding in-charge will get the information through Cloud. For theft security process the toggle switch is used. It may be activated or deactivated the Theft security process. Ultrasonic sensor is used to produce the echo signal for stop the reproduction for animals (like cat, rat, etc) and lizard Ground agama, etc).

The Arduino takes the analog input form DHT sensor, Ultrasonic sensor and $\mathrm{Co} 2$ sensor and converts it into digital format.

The proposed system uses a sensor based monitoring using IOT which maintains the temperature, humidity and keeps away the insects from the food grains and also avoids the usage of pests.
Revised Manuscript Received on October 12, 2018.

Kavitha D, Department of Computer Science Engineering, Sri Sairam Engineering College, Chennai

Anusha R, Deepa Lakshmi V, Mythili N, Department of Computer Science Engineering, Sri Sairam Engineering College, Chennai.

Shali A, Department of Computer Science Engineering, Sri Sairam Engineering College, Chennai 


\section{Smart System for Efficient Monitoring of Food Grains}

A. Architecture Diagram

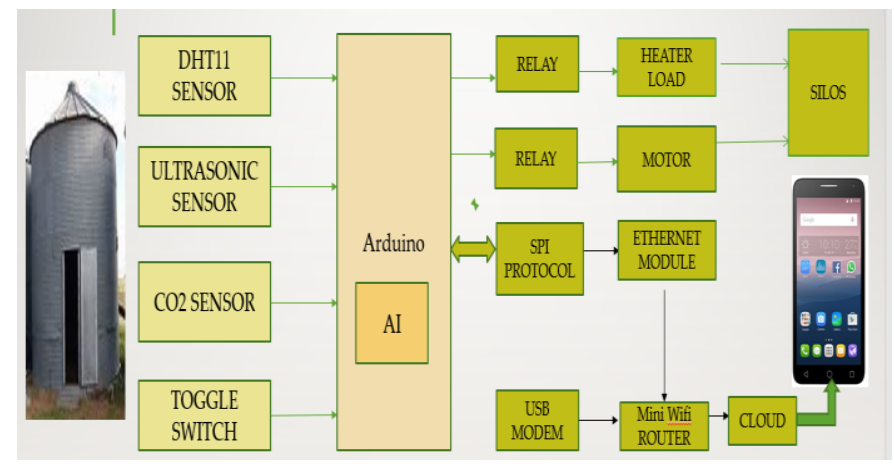

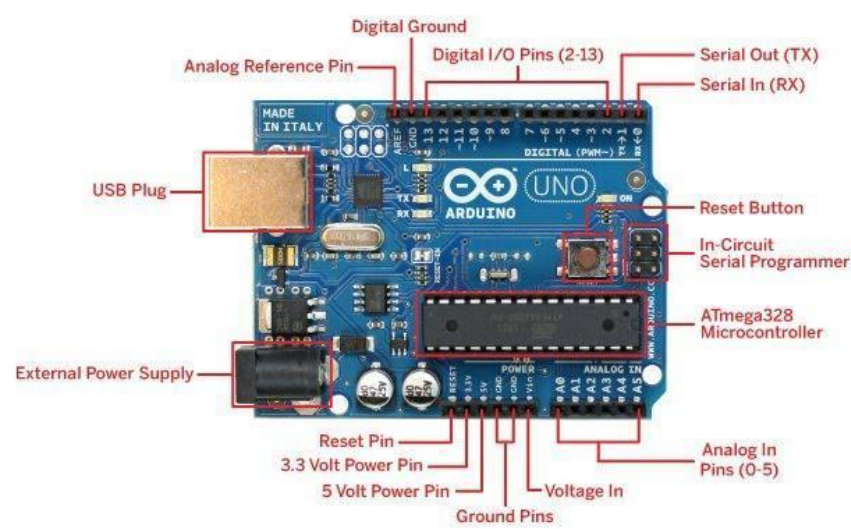

\section{Cloud Interface With Arduino}

The Arduino generated data, sends to SPI protocol. Four wires of SPI protocol such as MOSI, CLK, SS and MISO used for master/slave communication.

The master is a microcontroller, and the slaves are other

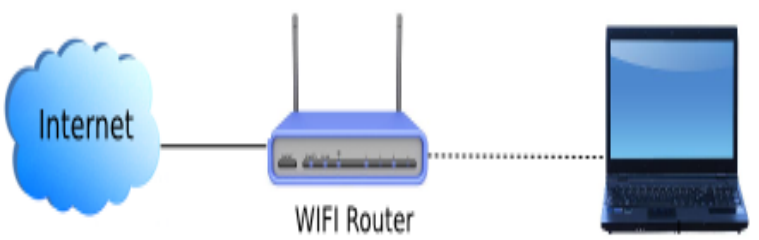

peripherals like sensors, GPS modem and GSM modem etc. Through SPI serial bus the multiple slaves are interfaced with the master. It is then transmitted to ethernet module which connects to the cloud through ethernet cable.

\section{Application Development}

Data from the cloud are then transmitted to the application which raises an alert if any insects population and theft occurs in the silos and also messages transmitted to the silos incharge.

\section{Assembling All Modules}

The sensor modules are integrated to the Arduino which converts analog signal to digital signal. These are further connected to motor and heater load through relays which converts the 5v DC signal of the Arduino to 230V Ac signal for processing . The RTOS cloud system which is connected to mini Wifi router for transmitting the data to the application. 


\section{RESULTS AND OUTPUTS}

\section{A. Grain Monitoring}

The grains stored in the storage units are monitored using multiple sensors and the data is processed by the microcontroller i.e., Arduino and then it is sent to the user through Ethernet. Here we use temperature sensor to sense the temperature of the grains because temperature has direct effect on grain conditions, humidity sensor is used to sense the amount of moisture present in the air because too much moisture would spoil the grains which are stored and also $\mathrm{CO} 2$ sensor is also used to monitor $\mathrm{CO} 2$ levels because there is a great risk of $\mathrm{CO} 2$ changing into $\mathrm{CO}$ i.e., carbon monoxide which is a harmful gas that can even cause death.

\section{B. Grain Conditioning}

The grains stored if happen to meet adverse conditions then it is controlled by various steps as mentioned below. If the temperature is increased then a fan is switched on through a relay and when the temperature reaches the normal value then the fan is switched off automatically by sensing it using sensor and in the same way when the humidity increases a light is switched on till the value becomes normal. And this system also ensures security byusing a hidden door which closes automatically when unauthorized person enters. By using all these steps the grain conditions are controlled and maintained.

\section{C.Simulation Output}

The simulated output is generated by using Proteus software. Proteus Design Suite is a software tool suite used primarily for electronic design automation.

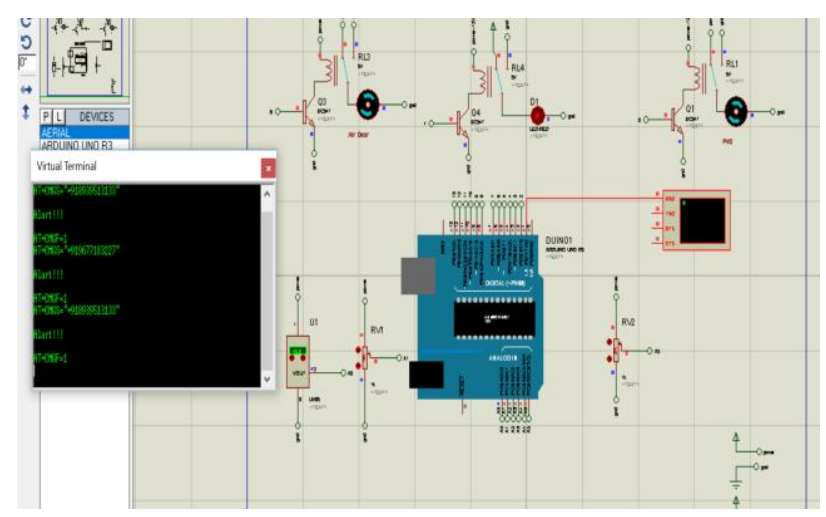

The lower machine control unit system is based on embedded Arduino. The DHT11-sensor stores temperature and humidity details. If the Humidity level is high automatically fan will rotate. At the same time corresponding in charge will get the information through Cloud.
The level of grain situation based on database is determined by the superior machine ARDUINO, and the PH3 gas is sprayed to kill the grain insects.

Toggle switch is used for activate or deactivate the Theft security process. Ultrasonic sensor is used to produce the echo signal for stop the reproduction for animals (like cat, rat, etc) and lizard Ground agama, etc). And also it is used to detect the person entry in the store. Here we are designed one hidden door for security purpose. If the person entry to the store automatically the information is send to the corresponding in charge.

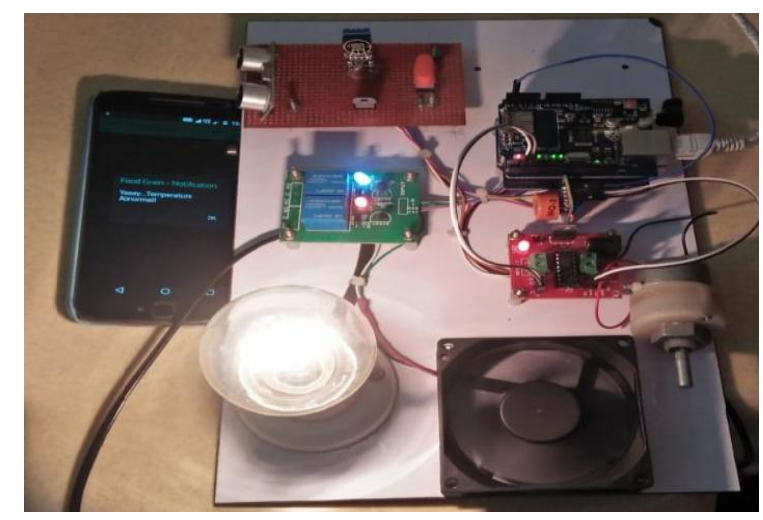

IV. CONCLUSION

The Design implementation of the system provides security and workability, flexibility and the ability to be easily carrying the data transmission through the network with minimum power usage. To maintain and controlled atmospheres or airtight storage domain for controlling of insect growth in the effective storage system and it is also used for bulk storage. To provide grain security and safety in grain storage environment, some of the measuring techniques of insecticides are used to control and monitor the insect population.

\section{REFERENCES}

1. Bin Liu Maryam Rezaei "Food loss and waste in the food supply chain," Food and Agriculture Organisation of the United Nations. July 2017.

2. Nagarajan V and Rajasekaran A International Journal of Applied Engineering Research, "Improved cluster head selection for energy efficient data aggregation in sensor networks," vol. 11, 2016.

3. Mythili Thirugnanam, Amol Bandal "Quality measurements offruits and vegetables using sensor network," Big Data and Cloud Computing Challenges(ISBCC-16 Š). Springer, 2016, pp. 121-130.

4. M.S .Anap, S.G Galande, "A Parameter Monitoring and Control of Grain Storage by Embedded System". International Journal of Informative and Futuristic Research, 2(11), 4172-4179.

5. Ahmed Toman ,Mabrouk, and, Aya Abdelmonsef, "Smart grain storage monitor and control," American Scientific Research Journal for Engineering, Technology, and Sciences, vol. 31, no. 1, pp. 156-162, 2017.

6. Jie Zhang, Xiaodong Zhang, and Xiujuan Li, "Design and implementation of embedded monitoring system for grain storage," in Information Management and Engineering (ICIME), 2010, pp. 197-200. 


\section{AUTHORS PROFILE}

D.KAVITHA M.Tech(CSE), Assistant Professor, Department of Computer Science and Engineering, Sri Sairam Engineering College having 5+ years of teaching experience . My area of interest is Networking and Distributed Systems.

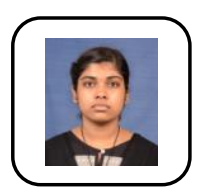

R.ANUSHA, BE(CSE), Student, Department of Computer Science and Engineering, Sri Sairam Engineering College. CSI Member. My Area of interest IOT and Data Mining.

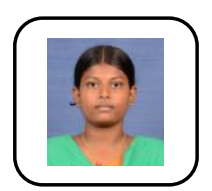

V.DEEPALAKSHMI , BE(CSE), Student, Department of Computer Science and Engineering, Sri Sairam Engineering College. CSI Member. My Area of interest IOT , Machine Learning.

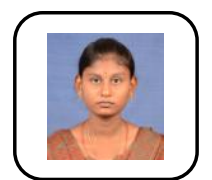

N.MYTHILI, BE(CSE), Student, Department of Computer Science and Engineering, Sri Sairam Engineering College. CSI Member. My Area of interest IOT, Machine Learning and Computer Vision.

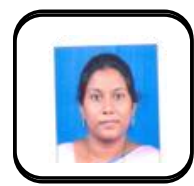

A.SHALI B.E.,M.E(CSE), Assistant Professor, Department of Computer Science and Engineering, Sri Sairam Engineering College having 10+ years of teaching experience. My area of interest is Big Data Analytics and Cloud Computing. 